\title{
New Topological Configurations in the Continuous Heisenberg Spin Chain: Lower Bound for the Energy
}

\author{
Rossen Dandoloff \\ Laboratoire de Physique Théorique et Modélisation, Université de Cergy-Pontoise, 95302 Cergy-Pontoise, France \\ Correspondence should be addressed to Rossen Dandoloff; rossen.dandoloff@u-cergy.fr
}

Received 19 April 2015; Accepted 12 August 2015

Academic Editor: Oleg Derzhko

Copyright ( 2015 Rossen Dandoloff. This is an open access article distributed under the Creative Commons Attribution License, which permits unrestricted use, distribution, and reproduction in any medium, provided the original work is properly cited.

In order to study the spin configurations of the classical one-dimensional Heisenberg model, we map the normalized unit vector, representing the spin, on a space curve. We show that the total chirality of the configuration is a conserved quantity. If, for example, one end of the space curve is rotated by an angle of $2 \pi$ relative to the other, the Frenet frame traces out a noncontractible loop in $S O(3)$ and this defines a new class of topological spin configurations for the Heisenberg model.

\section{Introduction}

It is well known that the two-dimensional continuous Heisenberg model has very nice topological properties [1]. The order parameter is a normalized vector field $\mathbf{n}^{2}=1$ (therefore, the order parameter manifold is $S^{2}$ ). If we impose homogenous boundary conditions on the vector field $\mathbf{n}(\mathbf{r})_{r \rightarrow \infty}=\mathbf{n}_{0}$ (constant vector field), we can compactify the plane $R^{2}$ into $S^{2}$ and therefore the possible field configurations are classified by $\pi_{2}\left(S^{2}\right)=\mathbf{Z}$. The energy in each class is bounded from below $H \geq n J$, where $n$ is the number of times, $S^{2}$ is wrapped around $S^{2}$, and $J$ is the coupling constant in the Heisenberg spin Hamiltonian. These configurations are metastable because the Hamiltonian is symmetric under homothety: $H_{\lambda}=\int\left((\partial \mathbf{n} / \partial(\lambda x))^{2}+(\partial \mathbf{n} / \partial(\lambda y))^{2}\right) d \lambda x d \lambda y=H$ (where $\lambda$ is a real parameter). The one-dimensional Heisenberg model does not have this nice topological property. Under homogeneous boundary conditions, the line $R^{1}$ may be compactified to $S^{1}$, and now $\pi_{1}\left(S^{2}\right)=0$ and there are no different classes of configurations based on homotopy. In order to find out if there is a new topological structure in the one-dimensional case, one has to analyze the Heisenberg Hamiltonian in more detail. The vector field is normalized and therefore we will use the following representation for $\mathbf{n}=$ ( $\sin \theta \cos \phi, \sin \theta \sin \phi, \cos \theta$ ). Shankar [2] has found that for a finite two-dimensional system (e.g., of radius $R$ ) the situation is even worse; that is, all configurations are point-like. Now, we will concentrate on the finite one-dimensional case.

\section{Materials and Methods}

In $\theta$ and $\phi$ variables, the Hamiltonian has the form

$$
H=J \int_{-L}^{+L}\left(\theta_{s}^{2}+\sin \theta^{2} \phi_{s}^{2}\right) d s,
$$

where the subscript $s$ stands for $d / d s$ and $s$ denotes the coordinate along $R^{1}$. This Hamiltonian is not symmetric under homothety transformation $s \rightarrow \lambda s$ and therefore the spin configurations are not metastable like in the $2 D$ case. The equations of motion for this spin Hamiltonian have been established [3] in taking $\phi$ and $\cos \theta$ to be the conjugated generalized coordinate and momentum so that the Poisson bracket gives $[\phi(x), \cos \theta(y)]=\delta(x-y)$. The generator of translations (momentum) is given by the following expression:

$$
P=\int_{-L}^{+L}(1-\cos \theta) \phi_{s} d s .
$$

It verifies the Poisson brackets: $[\phi(s), P]=-\phi_{s}$ and $[\cos \theta(s), P]=-(d / d s) \cos \theta(s)$. These relationships have first been proven for an infinite chain by Tjon and Wright [3]. $P$ is a constant of the motion. 


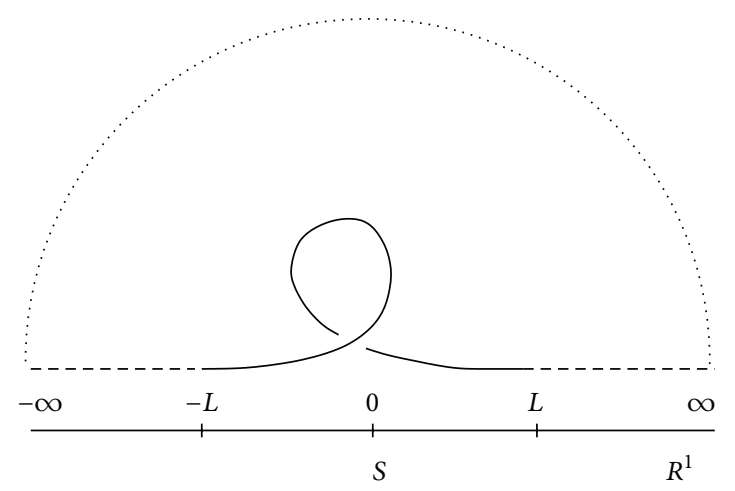

FIGURE 1: Spin configuration.

For our analysis of the possible spin configurations, it is useful to map the unit vector $\mathbf{n}$ to the unit tangent of a space curve [4]. Now, different space curves will represent different spin configurations. We will impose homogeneous boundary conditions; that is, spins at $\pm L$ will be parallel. Then, curves representing the different spin configurations will tend to the straight line as $s \rightarrow \pm L$. Here, we will concentrate on the geometrical and topological quantities characterizing these space curves. Of special interest for us will be the writhe of a curve (which characterizes the chirality of the curve). For a closed curve, it is defined as follows (see, e.g., [5]):

$$
W r=\frac{1}{4 \pi} \oint d s \oint d s^{\prime} \frac{\left(\mathbf{r}(s)-\mathbf{r}\left(s^{\prime}\right)\right) \cdot\left(\mathbf{n}(s) \times \mathbf{n}\left(s^{\prime}\right)\right)}{\left|\mathbf{r}(s)-\mathbf{r}\left(s^{\prime}\right)\right|^{3}}
$$

The tip of the radius vector $\mathbf{r}$ draws the curve, while $\mathbf{n}$ is the unit tangent. The writhe is properly defined on a ribbon or on a thin rod. After taking the width of the ribbon to zero, one gets the writhe for a closed curve. For the proper definition of the writhe of an open curve (where at both ends of the curve the tangents are parallel to each other), one has to complete the open curve by a closure at infinity which makes the combined curve a closed curve (see, e.g., Figure 1). We have to distinguish two classes of homotopically equivalent curves: one where two ends of the curve are rotated to each other by $2 \pi$ and the other where the two ends are rotated by $4 \pi[6,7]$. This last class includes the straight line (i.e., the ground state for the spin configuration). The first class of curves cannot be transformed continually into the ground state without crossing the curve itself. For this reason, we have to keep a memory of the triad defined on the ribbon [6]. These two classes are due to the fact that group $S O(3)$ is nonsimply connected manifold and closed loops in $\mathrm{SO}(3)$ fall into two classes: those that can be contracted to a point and those for which this is impossible. A triad evolving on the space curve from $s=-L$ to $s=L$ traces out a closed curve on $S O(3)$ (see, e.g., [8]).

A theorem by Fuller [9] allows one to express $W r$ as an integral of a local quantity. We will express $W r$ with respect to a reference curve $C_{0}[10]$ :

$$
W r=W r_{0}+\frac{1}{2 \pi} \int_{-L}^{+L} \frac{\mathbf{n}_{0} \times \mathbf{n} \cdot(d / d s)\left(\mathbf{n}_{0}+\mathbf{n}\right)}{\left(1+\mathbf{n}_{0} \cdot \mathbf{n}\right)} d s,
$$

where $W r_{0}$ is the writhe of the reference curve. The simplest choice is the straight line $C_{0}=(0,0, s)$ and then $\mathbf{n}_{0}=(0,0,1)$ and $W r_{0}=0$ [10]. A simple calculation gives the following expression for the writhe:

$$
W r=\frac{1}{2 \pi} \int_{-L}^{+L}(1-\cos \theta) \phi_{s} d s
$$

\section{Results and Discussion}

Our first observation is that the writhe $W r$ for the spin configurations (quantity that characterizes the chirality of the spin configuration) coincides with the total momentum $P$. The total momentum $P$ is a conserved quantity: it follows that $W r$ is a conserved quantity too. This will lead us to a new class of possible excitations for the continuous classical spin Heisenberg model. We will note first that the writhe $W r$ suffers discontinuity when one region of the curve crosses another and the jump is always +2 [5]. This means that all configurations that belong to the configuration of the ground state $(\theta=0$ for the real spins) are separated from all the other classes of configurations by a jump of the writhe $W r$. One configuration that does not belong to the ground state class of configurations is that represented by a space curve whose two ends are rotated by $2 \pi$ to each other. Let us consider one such configuration: the space curve representing the spin configuration goes from $-L$ to $+L$. This curve is completed by a straight line between $-L$ and $-\infty$ and between $+L$ and $+\infty$ and is closed by a semicircle at infinity in order to form a closed curve (see Figure 1). Note that on the straight segments and on the semicircle at infinity the curvature $k$ is zero. The writhe is zero for both straight segments when $s \in \pm(L, \infty)$ and for the infinite semicircle. This geometrical construction does not change the writhe of the actual curve. Such a curve belongs to a whole class of configurations which deform smoothly from one to another and are separated from the ground state class by a jump in the writhe $W r$. Belonging to this class of configurations will have consequences for the energy of the spin configuration too. It is well known that for a closed space curve [11]

$$
\oint k d s \geq 2 \pi
$$


Now, because the curvature $k \neq 0$ only for $s \in(-L,+L)$, the above inequality is equivalent to

$$
\int_{-L}^{+L} k d s \geq 2 \pi .
$$

Let us now consider the following Cauchy-Schwarz inequality:

$$
\left(\int_{-L}^{+L} k d s\right)^{2} \leq 2 L \int_{-L}^{+L} k^{2} d s
$$
[12]).

In Euler angles, the curvature $k^{2}=\theta_{s}^{2}+\sin ^{2} \theta \phi_{s}^{2}$ (see, e.g.,

Therefore, the energy of the spin chain satisfies the obvious inequality:

$$
\begin{gathered}
H=J \int_{-L}^{+L}\left(\theta_{s}^{2}+\sin ^{2} \theta \phi_{s}^{2}\right) d s=J \int_{-L}^{+L} k^{2} d s \\
\geq \frac{J\left(\int_{-L}^{+L} k d s\right)^{2}}{2 L} \geq J \frac{4 \pi^{2}}{2 L}=J \frac{2 \pi^{2}}{L} .
\end{gathered}
$$

\section{Conclusions}

The writhe $W r$ that characterizes the chirality of the spin configuration is a conserved quantity. The energy of the spin configuration is limited from below for this class of configurations (the ends of the space curve are rotated by $2 \pi$ to each other). Obviously, for an infinite chain $L \rightarrow \infty$, there is no more difference in the lower bounds for the energy in the two homotopy classes. On the other hand, the barrier which separates $P$ from the zeroth class remains. The above result is consistent with the inequality for the elastic energy of thin rod whose ends are rotated by $2 \pi$ relative to each other [8], where the result is based on the same property of the rotation group $S O(3)$. In this case, the thin rod has only bending rigidity $J$ and no torsional rigidity.

We have shown that there are topological configurations for the Heisenberg spin model even in the one-dimensional case.

\section{Conflict of Interests}

The author declares that there is no conflict of interests regarding the publication of this paper.

\section{References}

[1] A. A. Belavin and A. M. Polyakov, "Metastable states of twodimensional isotropic ferromagnets," JETP Letters, vol. 22, no. 10, p. 245, 1975.

[2] R. Shankar, "Applications of topology to the study of ordered systems," Journal de Physique Archives, vol. 38, no. 11, pp. 14051412, 1977.

[3] J. Tjon and J. Wright, "Solitons in the continuous Heisenberg spin chain," Physical Review B, vol. 15, no. 7, pp. 3470-3476, 1977.

[4] R. Balakrishnan, A. R. Bishop, and R. Dandoloff, "Geometric phase in the classical continuous antiferromagnetic Heisenberg spin chain," Physical Review Letters, vol. 64, no. 18, pp. 2107-2110, 1990.

[5] M. D. Frank-Kamenetskiü and A. V. Vologodskiü, "Topological aspects of the physics of polymers: the theory and its biophysical applications," Soviet Physics Uspekhi, vol. 24, no. 8, p. 679, 1981.

[6] G. H. M. van der Heijden, M. A. Peletier, and R. Planque, "On end rotation for open rods undergoing large deformations," Quarterly of Applied Mathematics, vol. 65, pp. 385-402, 2007.

[7] M. A. Berger and Ch. Prior, "The writhe of open and closed curves," Journal of Physics A, vol. 39, no. 26, pp. 8321-8348, 2006.

[8] J. C. Baez and R. Dandoloff, "Topological lower bound on the energy of a twisted rod," Physics Letters A, vol. 155, no. 2-3, pp. 145-147, 1991.

[9] F. B. Fuller, "Decomposition of the linking number of a closed ribbon: a problem from molecular biology," Proceedings of the National Academy of Sciences of the United States of America, vol. 75, no. 8, pp. 3557-3561, 1978.

[10] B. Fain, J. Rudnick, and S. Östlund, "Conformations of linear DNA," Physical Review E, vol. 55, no. 6, pp. 7364-7368, 1997.

[11] W. Fenchel, "Über krümmung und windung geschlossener raumkurven," Mathematische Annalen, vol. 101, no. 1, pp. 238252, 1929.

[12] R. Dandoloff and W. Zakrzewski, "Parallel transport along a space curve and relatedphases," Journal of Physics A: Mathematical and General, vol. 22, pp. L461-L466, 1989. 

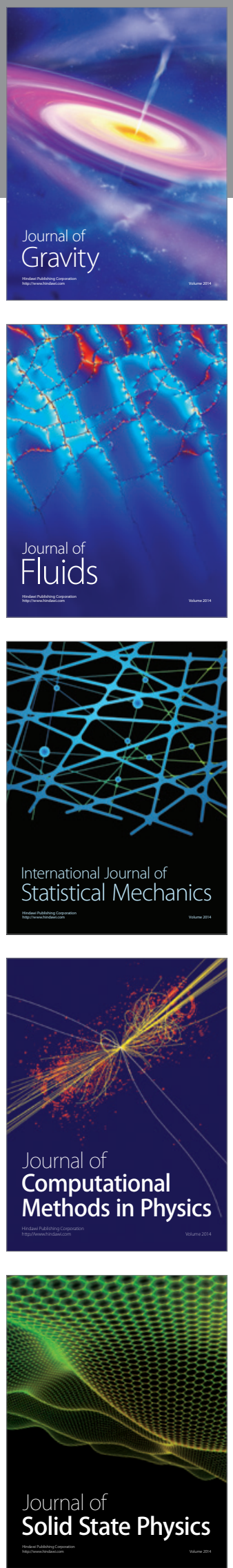

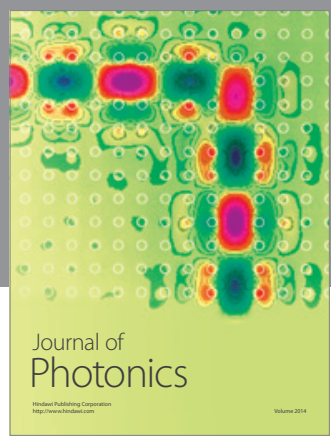

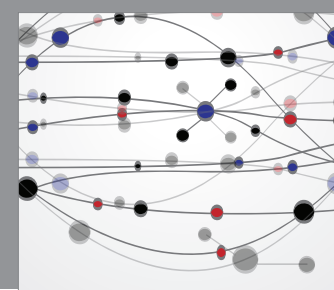

The Scientific World Journal

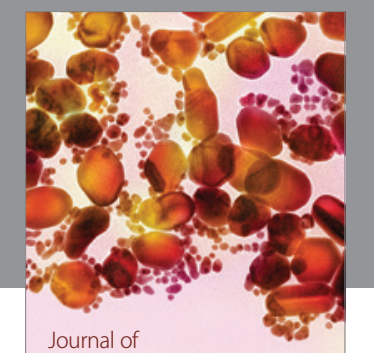

Soft Matter
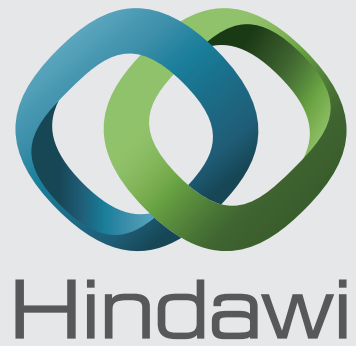

Submit your manuscripts at

http://www.hindawi.com
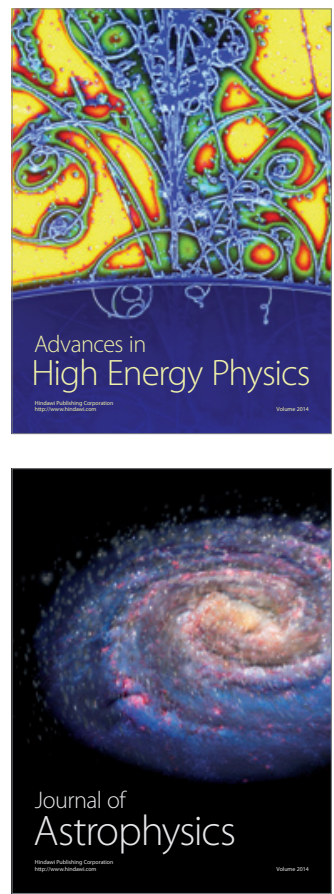
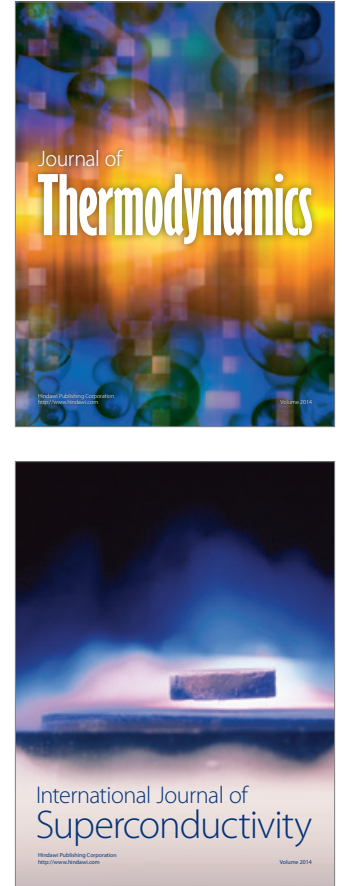
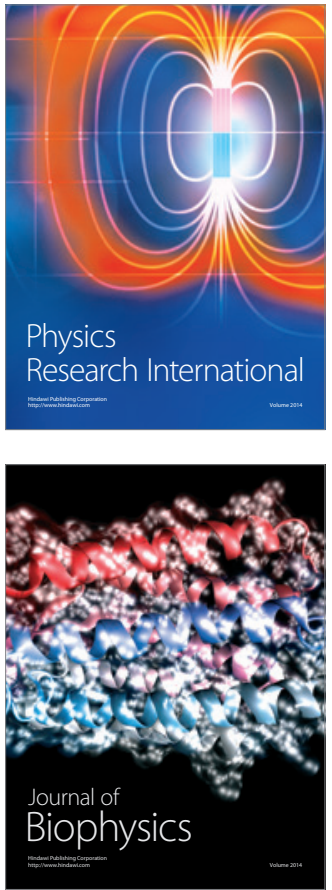
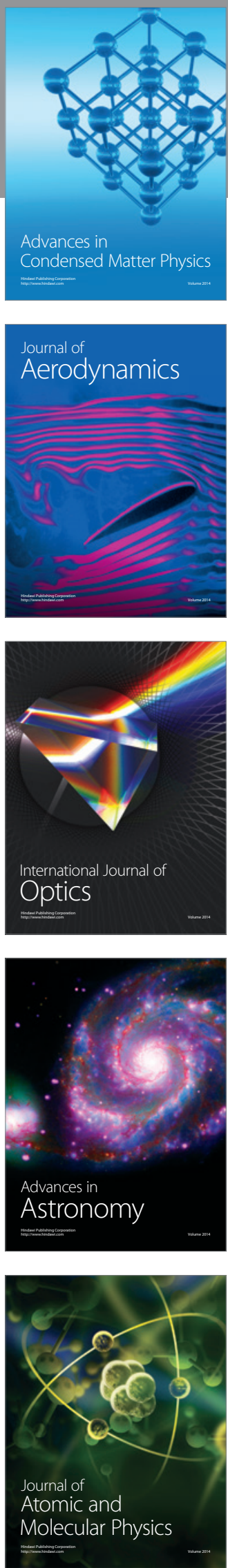